\title{
Las Tecnologías de la Información y Comunicación en el aprendizaje
}

\author{
Consuelo Belloch \\ Depto MIDE. Universidad de Valencia
}

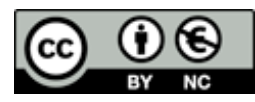

El gran desarrollo tecnológico que se ha producido recientemente ha propiciado lo que algunos autores denominan la nueva "revolución" social, con el desarrollo de "la sociedad de la información". Con ello, se desea hacer referencia a que la materia prima "la información" será el motor de esta nueva sociedad, y en torno a ella, surgirán profesiones y trabajos nuevos, o se readaptarán las profesiones existentes.

La dimensión social de las TIC se vislumbra atendiendo a la fuerza e influencia que tiene en los diferentes ámbitos y a las nuevas estructuras sociales que están emergiendo, produciéndose una interacción constante y bidireccional entre la tecnología y la sociedad. La influencia de la tecnología sobre la sociedad ha sido claramente explicitada por Kranzberg, en su ley sobre la relación entre tecnología y sociedad: "La tecnología no es buena ni mala, ni tampoco neutral" (1985: 50), pero esta relación no debe entenderse como una relación fatalista y determinista, sino que a nuestro entender nos conduce a nuevas situaciones y planteamientos que deben llevarnos a través de la investigación y el análisis de sus efectos a tomar posiciones que marquen el camino y la dirección a seguir atendiendo a la sociedad que deseamos construir. Los valores que dinamicen la sociedad serán los mismos que orienten el uso de las tecnologías, José Luis Sampedro en Técnica y globalización ${ }^{1}$ (2002), realiza una reflexión en profundidad sobre la globalización y la tecnología incidiendo en esta idea sobre la importancia de orientar su utilización para lograr una sociedad más humana, justa e igualitaria.

\section{Concepto de Tecnologías de la Información y Comunicación (TIC)}

Para Cabero las TIC: "En líneas generales podríamos decir que las nuevas tecnologías de la información y comunicación son las que giran en torno a tres medios básicos: la informática, la microelectrónica y las telecomunicaciones; pero giran, no sólo de forma aislada, sino lo que es más significativo de manera interactiva $e$ interconexionadas, lo que permite conseguir nuevas realidades comunicativas". (Cabero, 1998: 198)

\footnotetext{
${ }^{1}$ José Luis Sampedro (2002). Técnica y Globalización. Boletín Económico de ICE,, no 2750, 46. Disponible en http://www.clubcultura.com/clubliteratura/clubescritores/sampedro/miradas global.htm
} 
Podríamos definir las TIC como:

Tecnologías para el almacenamiento, recuperación, proceso y comunicación de la información

Existen múltiples instrumentos electrónicos que se encuadran dentro del concepto de TIC, la televisión, el teléfono, el video, el ordenador. Pero sin lugar a duda, los medios más representativos de la sociedad actual son los ordenadores que nos permiten utilizar diferentes aplicaciones informáticas (presentaciones, aplicaciones multimedia, programas ofimáticos,...) y más específicamente las redes de comunicación, en concreto Internet.

\section{Internet}

Podríamos definir Internet como la RED DE REDES, también denomina red global o red mundial. Es básicamente un sistema mundial de comunicaciones que permite acceder a información disponible en cualquier servidor mundial, así como interconectar y comunicar a ciudadanos alejados temporal o físicamente. Algunas de las características de la información de Internet han sido analizadas por Cabero (1998) como representativas de las TIC:

- Información multimedia. El proceso y transmisión de la información abarca todo tipo de información: textual, imagen y sonido, por lo que los avances han ido encaminados a conseguir transmisiones multimedia de gran calidad.

- Interactividad. La interactividad es posiblemente la característica más importante de las TIC para su aplicación en el campo educativo. Mediante las TIC se consigue un intercambio de información entre el usuario y el ordenador. Esta característica permite adaptar los recursos utilizados a las necesidades y características de los sujetos, en función de la interacción concreta del sujeto con el ordenador.

- Interconexión. La interconexión hace referencia a la creación de nuevas posibilidades tecnológicas a partir de la conexión entre dos tecnologías. Por ejemplo, la telemática es la interconexión entre la informática y las tecnologías de comunicación, propiciando con ello, nuevos recursos como el correo electrónico, los IRC, etc.

- Inmaterialidad. En líneas generales podemos decir que las TIC realizan la creación (aunque en algunos casos sin referentes reales, como pueden ser las simulaciones), el proceso y la comunicación de la información. Esta información es básicamente inmaterial y puede ser llevada de forma transparente e instantánea a lugares lejanos.

- Mayor Influencia sobre los procesos que sobre los productos. Es posible que el uso de diferentes aplicaciones de la TIC presente una influencia sobre los procesos mentales que realizan los usuarios para la adquisición de 
conocimientos, más que sobre los propios conocimientos adquiridos. En los distintos análisis realizados, sobre la sociedad de la información, se remarca la enorme importancia de la inmensidad de información a la que permite acceder Internet. En cambio, muy diversos autores han señalado justamente el efecto negativo de la proliferación de la información, los problemas de la calidad de la misma y la evolución hacia aspectos evidentemente sociales, pero menos ricos en potencialidad educativa -económicos, comerciales, lúdicos, etc.-. No obstante, como otros muchos señalan, las posibilidades que brindan las TIC suponen un cambio cualitativo en los procesos más que en los productos. Ya hemos señalado el notable incremento del papel activo de cada sujeto, puesto que puede y debe aprender a construir su propio conocimiento sobre una base mucho más amplia y rica. Por otro lado, un sujeto no sólo dispone, a partir de las TIC, de una "masa" de información para construir su conocimiento sino que, además, puede construirlo en forma colectiva, asociándose a otros sujetos o grupos. Estas dos dimensiones básicas (mayor grado de protagonismo por parte de cada individuo y facilidades para la actuación colectiva) son las que suponen una modificación cuantitativa y cualitativa de los procesos personales y educativos en la utilización de las TIC.

- Instantaneidad. Las redes de comunicación y su integración con la informática, han posibilitado el uso de servicios que permiten la comunicación y transmisión de la información, entre lugares alejados físicamente, de una forma rápida.

- Digitalización. Su objetivo es que la información de distinto tipo (sonidos, texto, imágenes, animaciones, etc.) pueda ser transmitida por los mismos medios al estar representada en un formato único universal. En algunos casos, por ejemplo los sonidos, la transmisión tradicional se hace de forma analógica y para que puedan comunicarse de forma consistente por medio de las redes telemáticas es necesario su transcripción a una codificación digital, que en este caso realiza bien un soporte de hardware como el MODEM o un soporte de software para la digitalización.

- Penetración en todos los sectores (culturales, económicos, educativos, industriales...). El impacto de las TIC no se refleja únicamente en un individuo, grupo, sector o país, sino que, se extiende al conjunto de las sociedades del planeta. Los propios conceptos de "la sociedad de la información" y "la globalización", tratan de referirse a este proceso. Así, los efectos se extenderán a todos los habitantes, grupos e instituciones conllevando importantes cambios, cuya complejidad está en el debate social hoy en día (Beck, U. 1999).

- Innovación. Las TIC están produciendo una innovación y cambio constante en todos los ámbitos sociales. Sin embargo, es de reseñar que estos cambios no siempre indican un rechazo a las tecnologías o medios anteriores, sino que en algunos casos se produce una especie de simbiosis con otros medios. Por ejemplo, el uso de la correspondencia personal se había reducido 
ampliamente con la aparición del teléfono, pero el uso y potencialidades del correo electrónico ha llevado a un resurgimiento de la correspondencia personal.

- Tendencia hacia automatización. La propia complejidad empuja a la aparición de diferentes posibilidades y herramientas que permiten un manejo automático de la información en diversas actividades personales, profesionales y sociales. La necesidad de disponer de información estructurada hace que se desarrollen gestores personales o corporativos con distintos fines y de acuerdo con unos determinados principios.

Algunas cuestiones a tener en cuenta en relación a la información que encontramos en Internet son:

- Es instantánea, podemos acceder a la misma generalmente de forma rápida y eficaz. La rapidez de acceso es de gran importancia para el usuario, una investigación realizada al respecto señaló que aquellas páginas web que tardaban en descargarse más de 20 segundos eran consideradas lentas por los usuarios y rechazadas, esto es no las volvían a visitar.

- Es dinámica y cambiante. Las informaciones que se encuentran en Internet se amplían todos los días. Además, las diferentes páginas son dinámicas cambiando su contenido con cierta asiduidad. Tanto es así, que una de los criterios de calidad de la información es la mayor o menor actualización de la misma. Por ello, todas las páginas que ofrecen información y recursos deberían indicar la fecha en el que se realizó su última actualización.

Como hemos dicho, la información en Internet es 'temporal' de modo que aquella dirección electrónica que nos permitía acceder a un documento, en otro momento nos puede presentar otra información o producir un error. Por ello, cuando queramos referenciar o citar el documento debemos especificar 'Consultado en (poner fecha)'. Por ejemplo:

- Almerich, Gonzalo, Suárez, Jesús, Orellana, Natividad, Belloch Consuelo, Bo, Rosa y Gastaldo, Irene (2005). Diferencias en los conocimientos de los recursos tecnológicos en profesores a partir del género, edad y tipo de centro. Revista ELectrónica de Investigación y EValuación Educativa, v. 11, n. 2. http://www.uv.es/RELIEVE/v11n2/RELIEVEv11n2 3.htm

Consultado en (5-10-07).

- No toda la información que ofrece Internet es de libre acceso o gratuita. Aunque existen revistas especializadas on-line de libre acceso, estas coexisten con otras revistas que ofrecen resúmenes de los artículos y exigen suscripción o pago para la consulta del artículo completo. No obstante, sigue resultando una forma válida, flexible y rápida para disponer de información válida y actualizada. 
- Por último, la información en Internet puede ser 'engañosa' y responder a intereses particulares. En Internet podemos encontrar tanto información veraz y de calidad como 'seudoinformación' que bajo la apariencia de información de calidad oculta la finalidad de la misma que puede hacer que la información no sea totalmente veraz o esté sesgada hacia una dirección determinada. Por ello, es imprescindible cuando trabajamos con información obtenida a través de la red, contrastar dicha información con otros documentos y con el propio criterio del profesional. Disponer de direcciones electrónicas 'fiables', que hayan pasado filtros de calidad es importante para el profesional (ej. revistas científicas, asociaciones profesionales, información institucional,...).

Analizadas las características de la información en Internet, que consideramos relevantes para poder realizar un buen uso de la misma. Pasamos a detallar las herramientas que podemos utilizar para acceder a esta información de una forma rápida y eficaz.

\section{Evolución de Internet}

La web ha evolucionado desde su creación de forma rápida en diferentes aspectos:

- Rapidez de acceso y número de usuarios conectados.

- Ámbitos de aplicación. El uso de las redes de comunicación ha ido aumentando exponencialmente desde su creación, actualmente múltiples de las actividades cotidianas que realizamos se pueden realizar de forma más rápida y eficaz a través de las redes (reservas de hotel, avión, tren,..., pago de tributos, solicitud de cita previa, transferencias bancarias, compra electrónica, etc.).

- Tipo de interacción del usuario. La evolución que ha seguido la web en relación al rol que los usuarios tienen en el acceso a la misma ha ido también evolucionando.

Se conocen tres etapas en la evolución a Internet:

- Web 1.0. Se basa en la Sociedad de la Información, en medios de entretenimiento y consumo pasivo (medios tradicionales, radio, TV, email). Las páginas web son estáticas y con poca interacción con el usuario (web 1.0, páginas para

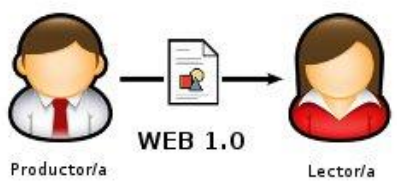
leer).

- Web 2.0. Se basa en la Sociedad del Conocimiento, la autogeneración de contenido, en medios de entretenimiento y consumo activo. En esta etapa las páginas web se caracterizan por ser dinámicas e interactivas (web 2.0, páginas para leer y

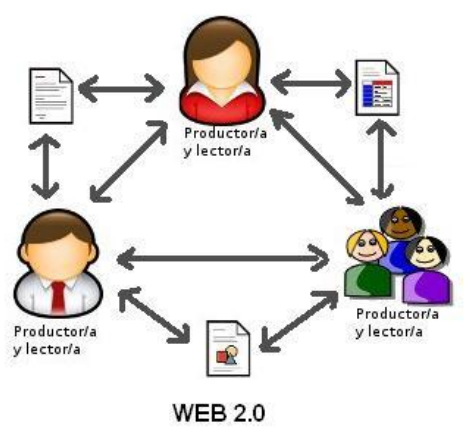


escribir) en donde el usuario comparte información y recursos con otros usuarios.

Algunas de las herramientas desarrolladas han permitido:

- Establecer redes sociales que conforman comunidades en donde los usuarios pueden incluir sus opiniones, fotografías, y comunicarse con el resto de miembros de su comunidad, Por ejemplo: MySpace, Facebook, Tuenti.

- Compartir y descargar diferentes tipos de recursos.

- imágenes: Flick-r

- videos: Youtube

- libros: Google books

- Facilitar la participación y colaboración.

- Documentos colaborativos: Wikis

- Páginas personales. Blogs

- $\quad \ldots$

- Web 3.0. Las innovaciones que se están produciendo en estos momentos se basan en Sociedades Virtuales, realidad virtual, web semántica, búsqueda inteligente.

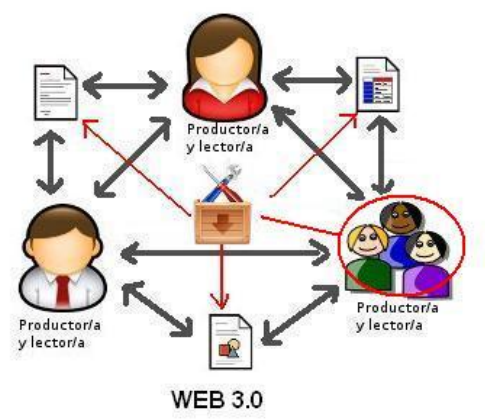

\section{Integración de las TIC en la Educación}

La sociedad de la información en la que estamos inmersos requiere nuevas demandas de los ciudadanos y nuevos retos a lograr a nivel educativo. Entre ellos:

- Disponer de criterios y estrategias de búsqueda y selección de la información efectivos, que permitan acceder a la información relevante y de calidad.

- El conocimiento de nuevos códigos comunicativos utilizados en los nuevos medios.

- Potenciar que los nuevos medios contribuyan a difundir los valores universales, sin discriminación a ningún colectivo.

- Formar a ciudadanos críticos, autónomos y responsables que tengan una visión clara sobre las transformaciones sociales que se van produciendo y puedan participar activamente en ellas.

- Adaptar la educación y la formación a los cambios continuos que se van produciendo a nivel social, cultural y profesional.

Las Tecnologías de la Información y Comunicación (TIC) han ido integrándose en los centros educativos de forma paulatina. A las primeras reflexiones teóricas que los profesionales de la educación realizaban sobre la adecuación o no de estas tecnológicas para el aprendizaje, se ha continuado con el análisis sobre el uso de estas tecnologías y su vinculación a las teorías de aprendizaje, junto a propuestas metodológicas para su implementación. 
El uso de las TIC no conduce necesariamente a la implementación de una determinada metodología de enseñanza/aprendizaje. Se producen en múltiples ocasiones procesos educativos que integran las TIC siguiendo una metodología tradicional en la que se enfatiza el proceso de enseñanza, en donde el alumno recibe la información que le trasmite el profesor y en la que se valoran fundamentalmente la atención y memoria de los estudiantes. No obstante, los profesores que deseen guiar los aprendizajes de sus alumnos, fomentando la interacción y el aprendizaje colaborativo siguiendo los postulados del constructivismo social de Vygostsky o el aprendizaje por descubrimiento de Bruner, tienen en las TIC un fuerte aliado, fundamentalmente en los diferentes recursos y servicios que ofrece Internet.

El impacto de las Tecnologías de la Información y Comunicación (TIC) sobre la educación, propicia posiblemente uno de los mayores cambios en el ámbito de la Educación. A través de Internet y de las informaciones y recursos que ofrece, en el aula se abre una nueva ventana que nos permite acceder a múltiples recursos, informaciones y comunicarnos con otros, lo que nos ofrece la posibilidad de acceder con facilidad a conocer personalidades de opiniones diversas. Por otro lado, las nuevas teorías de aprendizaje que centran su atención no tanto en el profesor y el proceso de enseñanza, como en el alumno y el proceso de aprendizaje, tienen un buen aliado en estos medios, si se utilizan atendiendo a los postulados del aprendizaje socioconstructivo y bajo los principios del aprendizaje significativo.

Como han demostrado diferentes estudios, el uso de las TICs en la educación depende de múltiples factores (infraestructuras, formación, actitudes, apoyo del equipo directivo, etc.), entre los cuales el más relevante es el interés y la formación por parte del profesorado, tanto a nivel instrumental como pedagógico. El estudio realizado por Apple Classrooms of Tomorrow (1985) en el que se analiza como integran los docentes los recursos tecnológicos (TIC), indica un proceso de evolución que sigue 5 etapas:

- Acceso: Aprende el uso básico de la tecnología.

- Adopción: Utiliza la tecnología como apoyo a la forma tradicional de enseñar.

- Adaptación: Integra la tecnología en prácticas tradicionales de clase, apoyando una mayor productividad de los estudiantes.

- Apropiación: Actividades interdisciplinares, colaborativas, basadas en proyectos de aprendizaje. Utilizan la tecnología cuando es necesaria.

- Invención: Descubren nuevos usos para la tecnología o combinan varias tecnologías de forma creativa.

\section{Las TICs en Pedagogía}

Los avances tecnológicos abren posibilidades de innovación en el ámbito educativo, que llevan a repensar los procesos de enseñanza/aprendizaje y a llevar a cabo un proceso continuo de actualización profesional.

La Pedagogía, al igual que otras disciplinas científicas, encuentra en las TIC nuevas actividades profesionales: 
- Análisis y evaluación de los recursos tecnológicos y su uso educativo.

- Integración de los medios de comunicación para lograr el aprendizaje.

- Diseño de estrategias educativas para favorecer la integración de recursos tecnológicos en diferentes ambientes de aprendizaje.

- Diseño de materiales multimedia para favorecer el proceso de enseñanza/aprendizaje.

- Desarrollo de materiales digitales.

- Diseño y evaluación de software educativo.

- Diseño, desarrollo y evaluación de modelos de educación presencial y a distancia.

- Diseño, aplicación y evaluación de los recursos tecnológicos.

- Planificación y diseño de cursos apoyados en la tecnología.

- Desarrollo, implementación y evaluación de cursos mediados por la tecnología.

\section{Referencias}

- Adell, J. (1996) Internet en educación: una gran oportunidad. Net Conexión, no11. Disponible en http://procesosemivirtual-ese.com/Internet en educacion una gran oportunidad.pdf

- Area, M. (2002) Sociedad de la Información, Tecnologías Digitales y Educación. Publicación on-line. Disponible en http://tecnologiaedu.us.es/bibliovir/pdf/tema1.pdf

- Bartolomé, A. (1996) Preparando para un nuevo modo de conocer. EDUTEC. Revista Electrónica de Tecnología Educativa, na 4. Disponible en http://www.uib.es/depart/gte/revelec4.html

- Beck, Ulrich (1999) What Is Globalization?. Cambridge: Polity Press.

- Cabero, J. (2005) Cibersociedad y juventud: la cara oculta (buena) de la Luna, en AGUIAR, M.V. y FARRAY, J.I. (2005): Un nuevo sujeto para la sociedad de la información. A Coruña, Netbjblo, 13-42. Disponible en http://tecnologiaedu.us.es/bibliovir/pdf/ciberiuve.pdf

- Rosique, R. Un asomo a la Educación: Web 1.0, 2.0 y 3.0, [online]. Disponible en http://www.monografias.com/trabajos71/asomo-educacion-web/asomoeducacion-web.shtml

- Salinas, J. (2001). TIC: ocupación y formación ¿globalización-desempleo?. CIFO III Congreso de Formación Profesional ocupacional. Formación, trabajo y certificación, 20 al 23 junio del 2001. Disponible en http://www.researchgate.net/publication/232242365 TIC ocupacin y forma cin globalizacin-desempleo/file/32bfe5100ea5c18c6e.pdf

- Sancho, J.Ma (Coord.)(2006) Tecnologías para transformar la educación. Ed. Alkal. Madrid. 
- Valzacchi, J.R. (2003) Internet y Educación: Aprendiendo y Enseñando en los Espacios Virtuales. AICD, INTERAMER DIGITAL 73. Disponible en http://www.educoas.org/Portal/bdigital/es/indice valzacchi.aspx?culture=es \&navid=201\&Highlight=true\&Search=VmFsemFjY2hp

\section{CITA:}

Belloch, C. (2012) Las Tecnologías de la Información y Comunicación en el aprendizaje. Material docente [on-line]. Departamento de Métodos de Investigación y Diagnóstico en Educación. Universidad de Valencia. Disponible en http://www.uv.es/bellochc/pedagogia/EVA1.pdf 